\title{
LAS: A combination of the analytic signal amplitude and the generalised logistic function as a novel edge enhancement of magnetic data
}

\author{
Luan Thanh $\mathrm{PHAM}^{1, *}$, Erdinc OKSUM${ }^{2}$, Thanh Duc DO ${ }^{1}$, \\ Minh LE-HUY ${ }^{3}$, Minh Duc VU ${ }^{1}$, Vinh Duc NGUYEN ${ }^{1}$ \\ ${ }^{1}$ Faculty of Physics, VNU University of Science, Vietnam National University, \\ Hanoi, Vietnam \\ ${ }^{2}$ Süleyman Demirel University, Engineering Faculty, Department of Geophysical Engineering, \\ Isparta, Turkey \\ ${ }^{3}$ Institute of Geophysics, Vietnam Academy of Science and Technology, \\ 18 Hoang Quoc Viet, Cau Giay, Hanoi, Vietnam
}

\begin{abstract}
In the evaluation of magnetic field data, edge enhancement and detection techniques are important treatments for the interpretation of geological structures. In general geological sense, contiguity of deep and shallow magnetic sources leads to weak and intense anomalies that complicates the interpretation to disclose adjacent anomalous sources. Many of the existing filters for edge detection in magnetics mostly have the disadvantage that they require a reduction to pole transformation as the pre-process of the data or they cannot balance weak and intense anomalies and therefore fail in detecting edges of deep and shallow sources simultaneously. This study presents an improved edge detection filter LAS (logistic function of the analytical signal), based on the generalised logistic function configured by the ratio of derivatives of the analytical signal. This novel approach has the capability of reducing the dependence on the direction of the magnetization and also balancing anomalies of sources at different levels of depth. The feasibility of the method is examined on both theoretical and real data cases comparatively with some other methods that utilize the analytical signal in their basis. In comparison, the results demonstrate that the LAS method provides more accurate estimation of edge localization.
\end{abstract}

Key words: generalised logistic function, analytic signal amplitude, edge detection

\section{Introduction}

The determination of the edges of the anomalous bodies is one of the important tasks in magnetic interpretation. The data usually contain the effect

\footnotetext{
*corresponding author: e-mail: luanpt@hus.edu.vn
} 
of sources located at different positions either in horizontal or vertical plane or the effects of sources with different magnetic properties, thus the overall anomaly mostly comprise weak and strong intensities together in the same frame. Most of existing edge detector filters are based on vertical and horizontal derivatives of the data. In this context, the 3D analytic signal (AS) (also called the total gradient) proposed by Roest et al. (1992) is one of the commonly used edge detection filter. Hsu et al. (1996) introduced an enhanced analytical signal $\left(\mathrm{AS}_{2}\right)$ that uses the higher order derivatives to reduce the interference effect due to adjacent sources.

Many previous works have interpreted the magnetic anomaly data using the AS and $\mathrm{AS}_{2}$ (e.g. Le-Huy et al., 2001, 2002; Salem and Ravat, 2003; Paoletti et al., 2004; Fairhead and Williams, 2006; Saleh and Pašteka, 2012; Saibi et al., 2016; Tran and Nguyen, 2017). However, both the AS and $\mathrm{AS}_{2}$ have limited achievement in simultaneously disclosing the edges from high and low amplitude anomalies. To balance the amplitudes of the different anomalies, normalized edge detector filters are used. Miller and Singh (1994) introduced the tilt angle that is defined as the arctangent of the ratio between vertical gradient and total horizontal gradient of the data. Verduzco et al. (2004) proposed the use of the total horizontal gradient of tilt angle. Wijns et al. (2005) suggested using the theta map method that is, in theory, the same angle as the tilt angle of the magnetic anomaly data. Cooper and Cowan (2006) suggested using horizontal tilt angle. Ferreira et al. (2013) introduced the tilt angle of the horizontal derivative amplitude. Zhang et al. (2014) used the tilt angle of the first order vertical gradient of the total horizontal gradient. Ma et al. (2014) used the tilt angle of the ratio between the first order horizontal gradient and the second order horizontal gradient. Yao et al. (2015) used the normalized enhanced analytic signal; Chen et al. (2017) used modified theta map filters. Nasuti and Nasuti (2018) proposed using a modified tilt angle that based on the vertical derivative of the analytical signal amplitude in different. Pham et al. (2019) suggested the use of the horizontal gradient amplitude configured in the generalised logistic function. However, although they can balance high and low amplitudes of anomalies, they still require pole reduction of the magnetic data (Li and Pilkington 2016; Nguyen et al., 2017; Pham et al., 2018; Pham et al., 2019). On the other hand, Ansari and Alamdar (2011) described the use of the analytic signal amplitude of the tilt angle 
(AT) which can be applied to the magnetic dataset directly. Although the method is more effective than the analytic signal, it still performs poorly in detecting all the edges of the body (Cooper, 2014a). Cooper (2014a) presented another approach namely the tilt angle of the amplitude of analytical signal (TAS) which is less dependent on the direction of the magnetization vector. In this study, we introduce an enhanced balanced edge detection filter that has reduced dependence on magnetization vector direction to improve edge detection.

The proposed filter utilises the generalised logistic function build up with the ratio of vertical derivative to total horizontal derivative of the AS. The potentiality of the proposed method is experienced on model anomalies of both $1 \mathrm{D}$ and $2 \mathrm{D}$ data and also on real magnetic anomalies from central India where the results are compared with other methods that realize the analytic signal amplitude and its modiefied versions.

\section{Method}

The generalised logistic function of the horizontal gradient amplitude is defined as (Nelder, 1961; Birch, 1999; Malkina-Pykh and Pykh, 2013; Pham et al., 2019):

LTHG $=\left[1+\exp \left(-\frac{\frac{\partial \mathrm{THG}}{\partial z}}{\sqrt{\left(\frac{\partial \mathrm{THG}}{\partial x}\right)^{2}+\left(\frac{\partial \mathrm{THG}}{\partial y}\right)^{2}}}\right)\right]^{-\alpha}$,

where THG is the total horizontal derivative of the magnetic intensity anomaly $M(x, y)$ :

$\operatorname{THG}(x, y)=\sqrt{\left(\frac{\partial M}{\partial x}\right)^{2}+\left(\frac{\partial M}{\partial y}\right)^{2}}$,

and $\alpha$ is a positive constant number decided by the interpreter. By Eq. (1), the method can equalize signals from different levels of depth. However, its practical application in magnetic interpretation still requires the pole reduction of the data. 
Here we suggest replacing THG by AS that is less dependent on the magnetization vector direction than the THG. Therefore, Eq. (1) becomes:

$$
\mathrm{LAS}=\left[1+\exp \left(-\frac{\frac{\partial \mathrm{AS}}{\partial z}}{\sqrt{\left(\frac{\partial \mathrm{AS}}{\partial x}\right)^{2}+\left(\frac{\partial \mathrm{AS}}{\partial y}\right)^{2}}}\right)\right]^{-\alpha},
$$

where

$$
\mathrm{AS}=\sqrt{\left(\frac{\partial M}{\partial x}\right)^{2}+\left(\frac{\partial M}{\partial y}\right)^{2}+\left(\frac{\partial M}{\partial z}\right)^{2}} .
$$

Following Florio et al. (2006), the analytic signal is a nonharmonic function, so its vertical derivative cannot be computed by conventional methods. The direct expressions for the derivatives of the analytic signal amplitude are given by Cooper (2014b) as:

$$
\begin{gathered}
\frac{\partial \mathrm{AS}}{\partial x}=\frac{\frac{\partial M}{\partial x} \frac{\partial^{2} M}{\partial x^{2}}+\frac{\partial M}{\partial y} \frac{\partial^{2} M}{\partial x \partial y}+\frac{\partial M}{\partial z} \frac{\partial^{2} M}{\partial x \partial z}}{\mathrm{AS}} \\
\frac{\partial \mathrm{AS}}{\partial y}=\frac{\frac{\partial M}{\partial x} \frac{\partial^{2} M}{\partial x \partial y}+\frac{\partial M}{\partial y} \frac{\partial^{2} M}{\partial y^{2}}+\frac{\partial M}{\partial z} \frac{\partial^{2} M}{\partial y \partial z}}{\mathrm{AS}} \\
\frac{\partial \mathrm{AS}}{\partial z}=\frac{\frac{\partial M}{\partial x} \frac{\partial^{2} M}{\partial x \partial z}+\frac{\partial M}{\partial y} \frac{\partial^{2} M}{\partial y \partial z}+\frac{\partial M}{\partial z} \frac{\partial^{2} M}{\partial z^{2}}}{\mathrm{AS}}
\end{gathered}
$$

The enhancement by this configuration reduces the dependence on the direction of the magnetization but also balances anomalies from different levels of depth and produces maximal amplitudes on the edges of source body.

This filter uses the ratio of derivatives of the amplitude of analytic signal to display the edges of strong and weak amplitude simultaneously. The main attributes of our edge detector filter are to provide maximum peak values on the edges of source body, as well as to reduce the dependence on the direction of the magnetization and to balance anomalies from shallow and deep sources. 


\section{Methods used for comparison}

Four different methods based on analytical signal which can be applied directly to the magnetic data set have been chosen to show comparatively the effectiveness of the LAS method. They are the analytical signal (AS) (Eq. (4)), the enhanced analytical signal $\left(\mathrm{AS}_{2}\right)$, the analytical signal amplitude of the tilt angle (AT) and the tilt angle of the ratio of derivatives of the analytic signal amplitude (TAS).

The $\mathrm{AS}_{2}$ is based on the higher order derivatives to enhance the source edges. This filter is given by Hsu et al. (1996):

$\mathrm{AS}_{2}=\sqrt{\left(\frac{\partial M_{z z}}{\partial x}\right)^{2}+\left(\frac{\partial M_{z z}}{\partial y}\right)^{2}+\left(\frac{\partial M_{z z}}{\partial z}\right)^{2}}$

where $M_{z z}$ is the second order derivative of the magnetic intensity anomaly.

Ansari and Alamdar (2011) used the analytic signal amplitude of the tilt angle. Its expression is:

$\mathrm{AT}=\sqrt{\left(\frac{\partial T}{\partial x}\right)^{2}+\left(\frac{\partial T}{\partial y}\right)^{2}+\left(\frac{\partial T}{\partial z}\right)^{2}}$,

where:

$T=\operatorname{atan}\left(\frac{\frac{\partial M}{\partial z}}{\sqrt{\left(\frac{\partial M}{\partial x}\right)^{2}+\left(\frac{\partial M}{\partial y}\right)^{2}}}\right)$.

Cooper (2014a) suggested the use of the arctangent function of the ratio of derivatives of the analytic signal expressed as:

$\operatorname{TAS}=\operatorname{atan} \frac{\frac{\partial \mathrm{AS}}{\partial z}}{\sqrt{\left(\frac{\partial \mathrm{AS}}{\partial x}\right)^{2}+\left(\frac{\partial \mathrm{AS}}{\partial y}\right)^{2}}}$.

\section{Synthetic examples}

In this section, the proposed method is applied to $2 \mathrm{D}$ and $3 \mathrm{D}$ synthetic examples to evaluate its effectiveness. The results of the present method 
were compared with the results of $\mathrm{AS}, \mathrm{AS}_{2}$, AT and TAS methods. In the 2-D example, the above methods are applied to magnetic anomalies (Fig. 1b) due to a 2D block (Fig. 1a) for a range of geomagnetic field inclinations $\left(0^{\circ}, 30^{\circ}, 60^{\circ}\right.$, and $\left.90^{\circ}\right)$. Figs. $1 \mathrm{c}, \mathrm{d}, \mathrm{e}$ and $\mathrm{f}$ display the outputs of $\mathrm{AS}, \mathrm{AS}_{2}$,
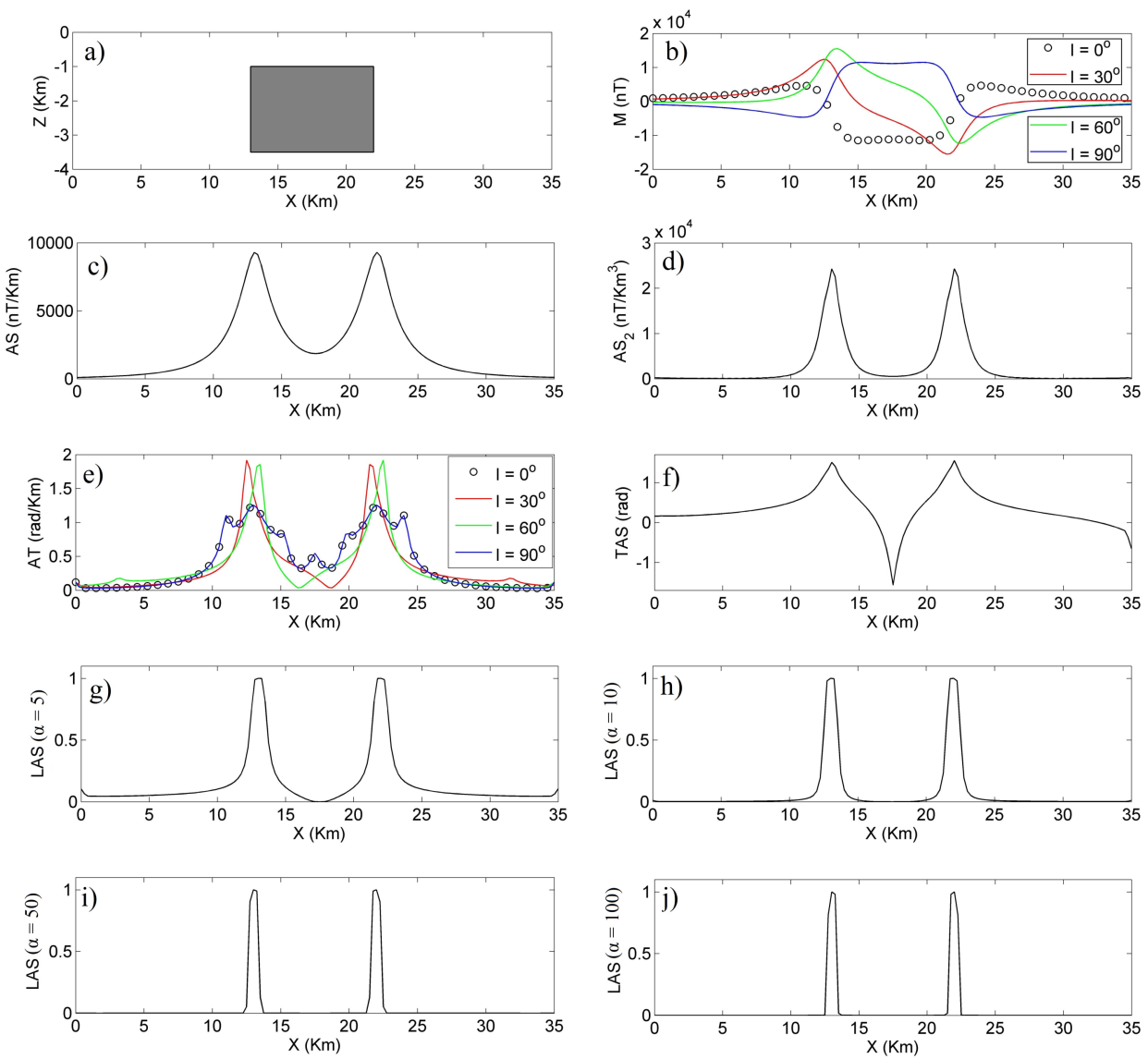

Fig. 1. (a) 2D block model (gray shading); (b) Total magnetic intensity M across a 2D block (Fig. 1a) with varying inclinations; (c) The analytic signal amplitude AS for all varieties of inclinations; (d) The enhanced analytic signal $\mathrm{AS}_{2}$ for all varieties of inclinations; (e) The analytic signal amplitude of the tilt angle AT for all varieties of inclinations; (f) The tilt angle of the analytic signal amplitude TAS for all varieties of inclinations; (g) The generalised logistic function of the analytic signal amplitude LAS for all varieties of inclinations, with $\alpha=5$; (h) with $\alpha=10$; (i) with $\alpha=50$; (j) with $\alpha=100$. 
AT and TAS applied to the data given in Fig. 1b. The maxima of the $\mathrm{AS}, \mathrm{AS}_{2}, \mathrm{AT}$ and TAS can automatically recognise the edges of the source body. In this case, the $\mathrm{AS}, \mathrm{AS}_{2}$ and TAS are independent of magnetization direction, but for the AT, this independence is seem lost. Figs. 1g,h,i and $\mathrm{j}$ show the application of the proposed method with different values of the parameter $\alpha(5,10,50$ and 100). The LAS filter successfully detects the edges of the causative body when apply directly to the magnetic data, which clearly gives better resolution of the source edges than other filters, and can display the edges in a more centralized way. It can be observed from these figures that, as the value of the parameter $\alpha$ increase, the resolution of the edges of the sources also increases. Tests showed that $\alpha \geq 10$ yielded the best results.

The efficiency of the proposed method is also evaluated by two 3-D synthetic examples. The first 3D example involves a single prism with dimensions of $32 \times 32 \times 2 \mathrm{~km}^{3}$, at a depth of $2 \mathrm{~km}$, induced magnetization of $5 \mathrm{~A} / \mathrm{m}$, rotation angle of $30^{\circ}$, and a magnetization vector with an inclination of $30^{\circ}$ and a declination of $-1^{\circ}$. Fig. 2 illustrates the geometric appearance of the model. Fig. 3a shows the theoretical magnetic anomaly due to this model calculated by the forward formula of Rao and Babu (1991) on a field dimension of $63.5 \times 63.5 \mathrm{~km}$ with $0.5 \mathrm{~km}$ grid steps. The outlines of the body are shown by the black lines in planar views. Fig. $3 \mathrm{~b}$ is the AS and Fig. $3 \mathrm{c}$ is the $\mathrm{AS}_{2}$ of the magnetic data in Fig. 3a. It can be observed from these figures that the AS is not effective in balancing of high and low amplitude anoma-
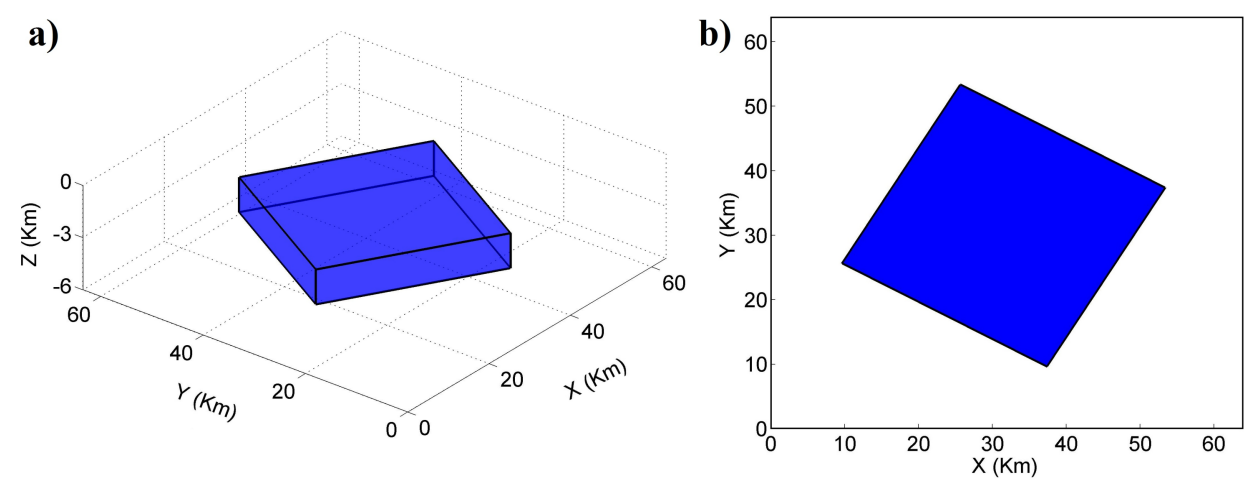

Fig. 2. (a) A 3D view of a synthetic model with one prism; (b) Plan view of the synthetic model in (a). 
lies simultaneously. Although the $\mathrm{AS}_{2}$ has a better resolution than the AS, similar shortcomings can also be observed for this as well. Fig. 3d shows the AT of the magnetic anomaly data in Fig. 3a. The AT is more effective than the $\mathrm{AS}$ and $\mathrm{AS}_{2}$ in balancing all the edges of the source body, but although the maximal values of AT can enclose the borders of the source, estimation of edges near to the corners are diffused. Figs. 3e and f show the TAS and LAS of the magnetic data in Fig. 3a, respectively. Although the TAS can enhance all the edges of the source body, the identified edges is lower in resolution. Besides, the maximum values of LAS delineates the edges of the real model more in detail. Consequently, by the overall comparison of the edge detection results obtained from the four filters applied in this example, the LAS filter achieves higher resolution of the edges than the others.
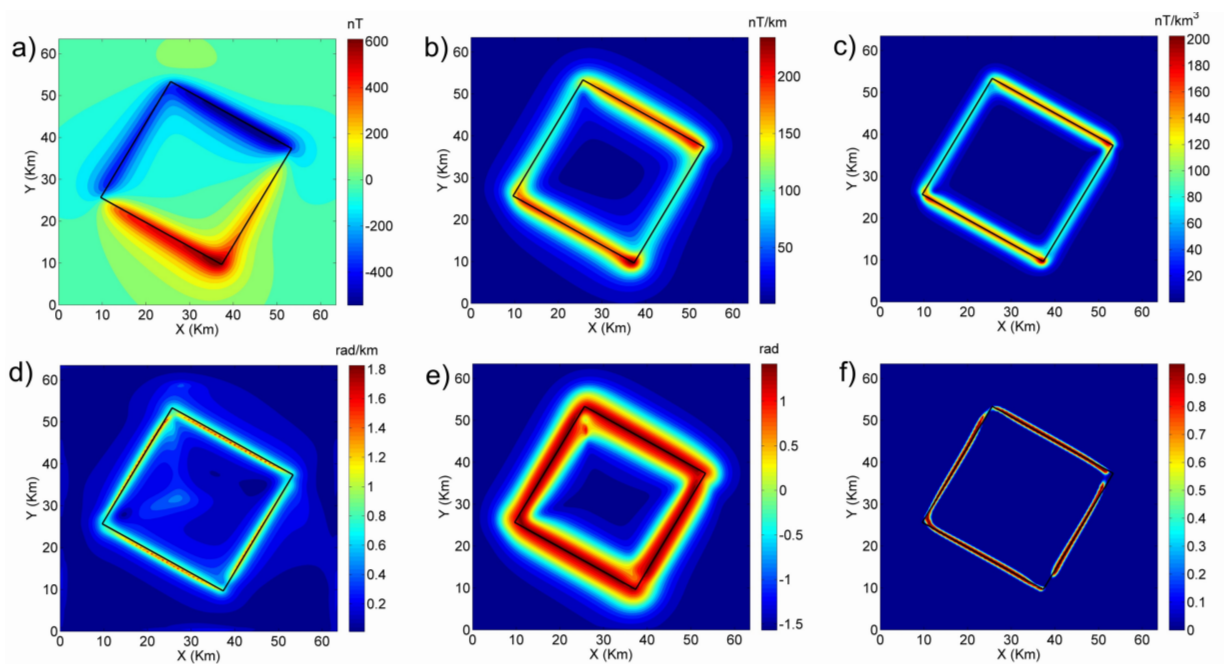

Fig. 3. (a) Synthetic magnetic anomaly of the single prism model; (b) AS; (c) AS2; (d) AT; (e) TAS; (f) LAS; with $\alpha=50$.

The second 3D example involves three prisms same in size but located at different depths and also with varied low magnetic inclinations (Table 1). Fig. 4 illustrates the geometric appearance of the three prisms (A, B and C) model. Fig. 5a shows the magnetic anomaly due to the three prisms with addition of $0.2 \%$ Gaussian random noise in order to examine also the influence of noise in this example. The outlines in plan view of the bodies are shown by the black lines in all figures. Because the filters are defined 
Table 1. Parameters of the three prism model.

\begin{tabular}{lccc}
\hline Parameters / Prism ID & A & B & C \\
\hline Center coordinates $(\mathrm{km} ; \mathrm{km})$ & $12 ; 31.5$ & $31.5 ; 31.5$ & $51 ; 31.5$ \\
Inclination I $\left(^{\circ}\right)$ & 8 & 10 & 12 \\
Declination D $\left({ }^{\circ}\right)$ & 25 & 26 & 27 \\
Magnetization $(\mathrm{A} / \mathrm{m})$ & 5 & 5 & 5 \\
Length $\times$ Width $(\mathrm{km})$ & $45 \times 10$ & $45 \times 10$ & $45 \times 10$ \\
Depth of top $(\mathrm{km})$ & 1 & 2 & 3 \\
Depth of bottom $(\mathrm{km})$ & 2 & 3 & 4 \\
Rotation angle $\left(^{\circ}\right)$ & 0 & 0 & 0 \\
\hline
\end{tabular}

by derivatives which increase the noise influence, upward continuation filter is applied prior to calculations of edge detection. Fig. 5b,c,d,e and $\mathrm{f}$ display the results of the AS, $\mathrm{AS}_{2}$, AT, TAS and LAS after upward continuation of $0.2 \mathrm{~km}$, respectively. As can be seen from Fig. $5 \mathrm{~b}$, in this low-inclination case, the AS is only effective in delineating two of the four edges of each causative body. It is worth noting that the $\mathrm{AS}_{2}$ uses third order derivatives of the anomalies while the others use first or second order derivatives, thus, this filter is more sensitive to noise. In this case, the $\mathrm{AS}_{2}$ is only effective in enhancing two of the four edges of shallow source (body A), and fails to detect any edges from the deeper sources (body B and C) (Fig. 5c). Likewise, even though there are some offset from the actual edges, the edges of the shallow source are determined by the AT, but the edges associated with the deeper sources cannot be effectively identified by this filter (Fig. 5d). In comparison, the AT is clearly more sensitive to noise than the AS and
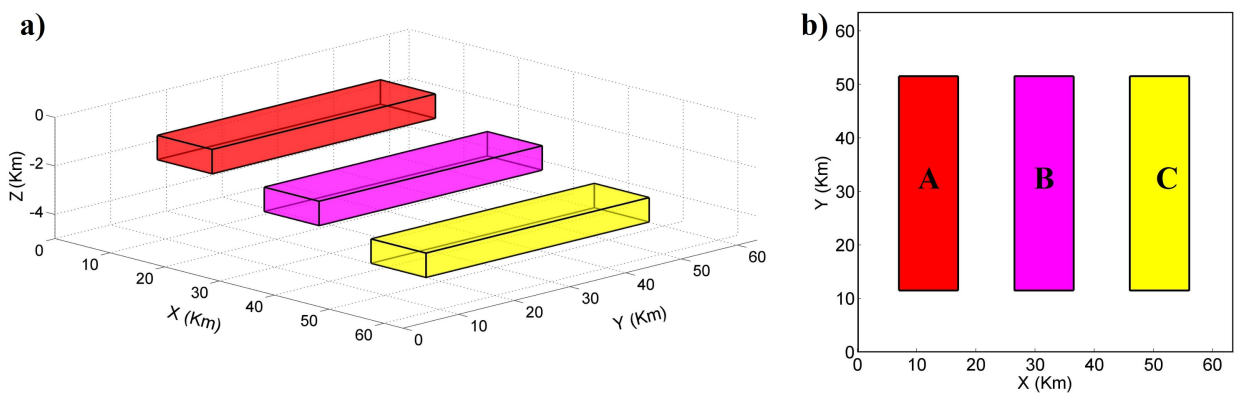

Fig. 4. (a) A 3D view of a synthetic model with three prisms; (b) Plan view of a synthetic model with three prisms. 

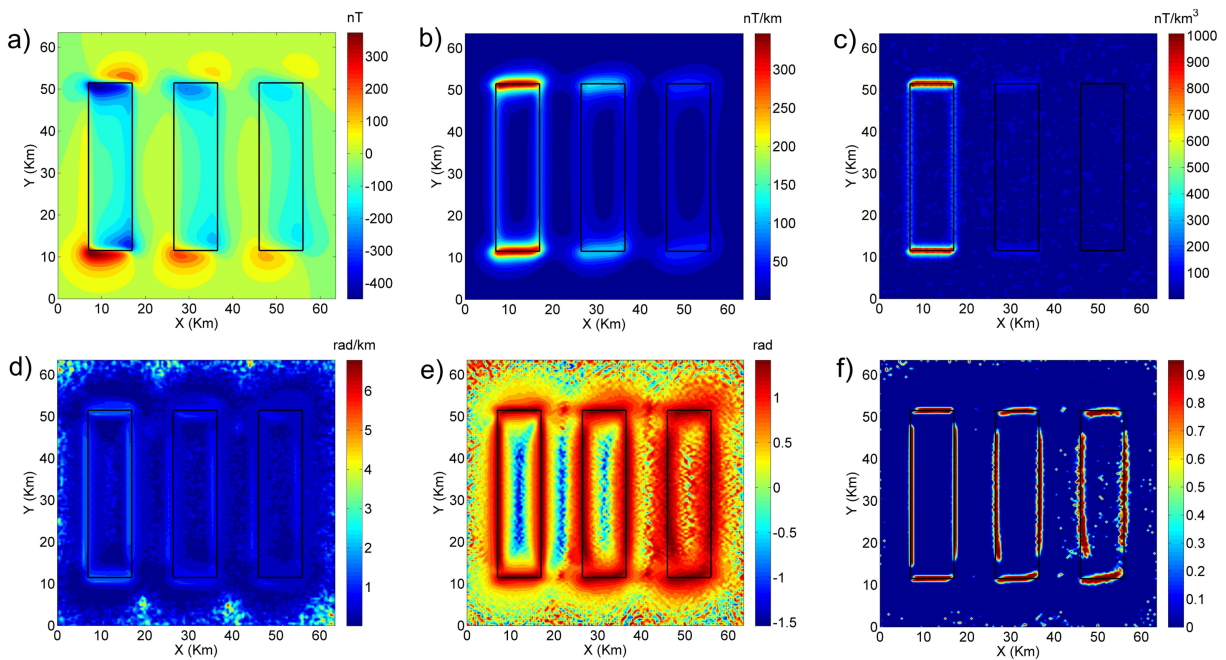

Fig. 5. (a) Synthetic magnetic anomaly of three prisms model; (b) AS; (c) AS2; (d) AT; (e) TAS; (f) LAS, with $\alpha=50$.

LAS. As can be seen from Fig. 5e and f, the TAS and LAS filters can enhance all the edges of the three causative bodies even they are located at different depths. Besides, although the results of the TAS and LAS methods produce similar results, it can be observed that LAS method discloses the true edges in higher resolution. Furthermore, it also takes advantage because the response of LAS values are nearly similar in amplitude with each other even the anomalies from the depth varied sources have different amplitudes. By comparison among the results in Fig. 5, it is apparent that the LAS results in enhance the edges to be more visible and the response is gained sharp over the true edges more than the AS, $\mathrm{AS}_{2}$, AT and TAS filters do.

\section{Real magnetic data application}

In this section, the practical applicability of the method proposed is demonstrated with the interpretation of magnetic anomaly data from the central part of the Indian continent (Fig. 6a). The study area lies between $80.49^{\circ} \mathrm{E}$ and $81.40^{\circ} \mathrm{E}$, of the eastern longitudes and $23.6^{\circ} \mathrm{N}$ and $24.35^{\circ} \mathrm{N}$, of northern latitudes, covering an area about $101 \mathrm{~km}$ by $83 \mathrm{~km}$. This area is located in a seismically active transition zone featured with varying geological complex- 
ities such as variation of density, magnetic susceptibility, seismic velocities, thermal regime, etc. (Ghosh, 2016). Fig. 6b shows the geological map of the area with different subsurface geological formation types, viz., Semri, Rewa, Bhander, Gondwana, Gneisses, Mahakoshal, Granite Plutons and Kaimur groups exist in the area with various ages of geological features (Mohanty, 2012; Ghosh, 2016; Chetty, 2017). A regional fault namely the Sone-Narmada fault is marked by the northern boundary of Mahakoshal groups ages (Mohanty, 2012). A number of subparallel east-northeast to west-southwest trending major shear zones dissecting the study area are also seen on the map.
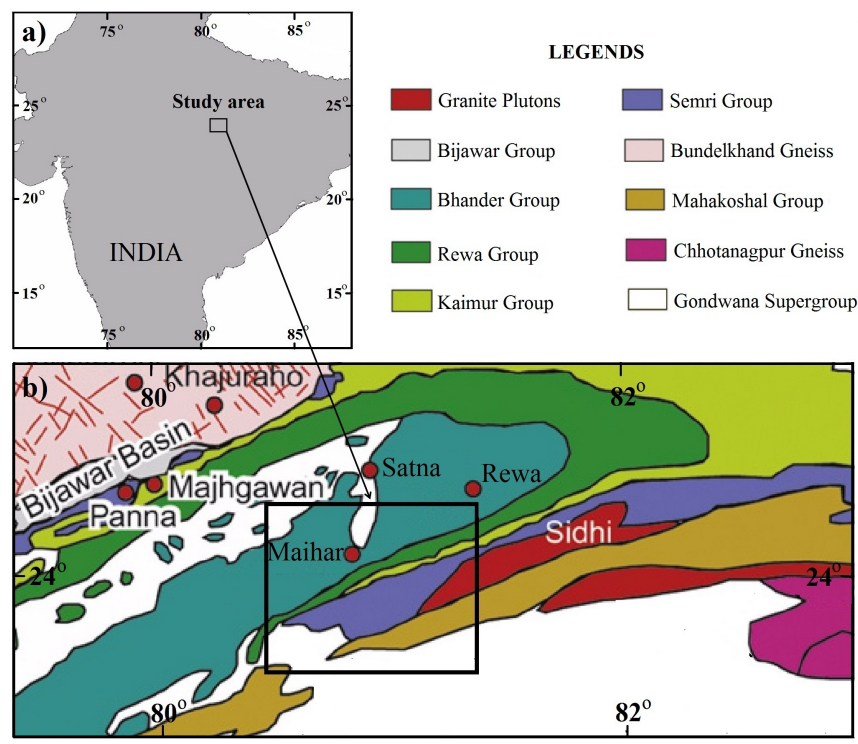

Fig. 6. Location and geological maps of the study area (modifed after Mohanty, 2012).

Fig. 7a shows the total field magnetic anomaly data of the study area (Ghosh, 2016) digitized on a $108 \times 66$ grid along the north-south and eastwest directions, respectively. Following Ghosh (2016), the magnetic data have been acquired by Geological Survey of India (Project Crumansonata, 1995). The data have been collected by Scintrex Fluxgate (MFM-2) magnetometer with the station interval of $1.0 \mathrm{~km}$. The data set vary from -813 to $953 \mathrm{nT}$ with anomalies having higher intensities locate over the northern part of the area, whereas anomalies having lower intensities appear in 

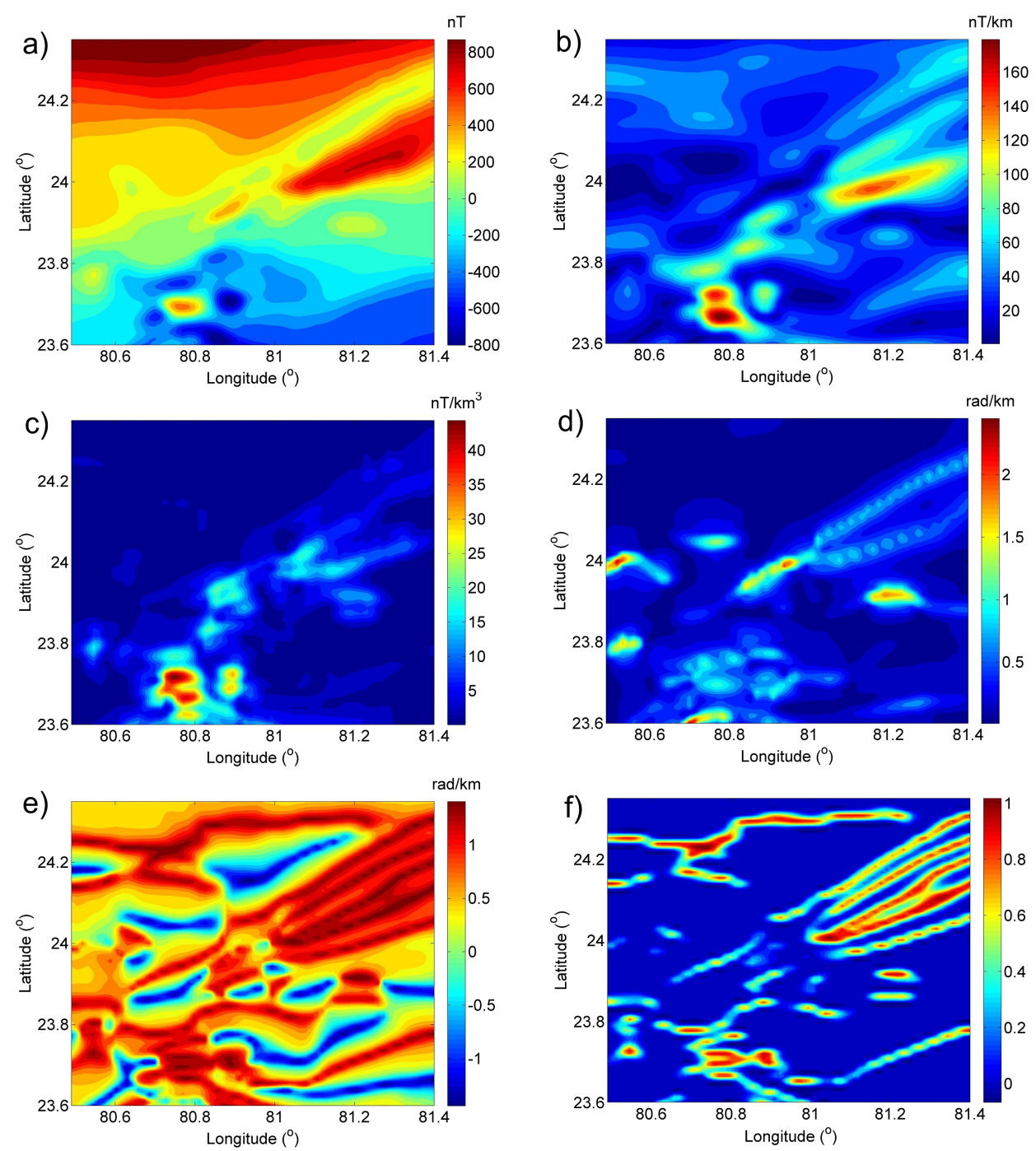

Fig. 7. (a) The total field magnetic anomaly of the study area; (b) AS; (c) AS2; (d) AT; (e) TAS; (f) LAS, with $\alpha=10$.

the southern part. The magnetic data trends in the area are varying eastnortheast to west-southwest direction. In order to attenuate the effects of shortest wavelenghts upward continuation to $2 \mathrm{~km}$ height is applied to the data set prior to determination of the edges. Hereby, this operation also 
produced results that are smoother and less sensitive to random noise than the original anomaly, but will not change the primary structures of the area. Fig. 7b,c,d and e show the AS, $\mathrm{AS}_{2}$, AT and TAS after upward continuation of magnetic dataset shown in Fig. 7a. It can be seen that the AS filter perform poor at balancing the edges from high and low amplitude anomalies simultaneously. As expected (and discussed in the above sections), the $\mathrm{AS}_{2}$ and $\mathrm{AT}$ results as more noisy than the other filters outputs and also give insufficient results to accurately determine the edges of the magnetic sources. The results from TAS reveals to be more effective in determining the source edges than the $\mathrm{AS}, \mathrm{AS}_{2}$ and $\mathrm{AT}$ but the recognized edges from this data are diffused as well.

Fig. $7 \mathrm{f}$ illustrates the LAS response of the upward continuated magnetic data. Both the TAS and LAS filters are less sensitive to noise than the $\mathrm{AS}_{2}$ and $\mathrm{AT}$, so can delineate more outlines than these methods. It can be observed from Figs. $7 b, c, d$,e and $f$ that the LAS is not only effective in balancing the different amplitude edges, but also provides the higher resolution, and can detect the edges of source body more prominently and precisely, making the geological structures more visible. By and large, as can be observed from Fig. 6b and Fig. 7f, there is a good correlation between the edges obtained from LAS filter and geologic structures, with many of structure trends are oriented in an east-northeast to west-southwest direction in the study area. However, some magnetic structures in the north-western part of this area are not identified by geological map alone (Fig. 6b) and their existence can only be detected through interpretation of the magnetic field data.

\section{Conclusions}

In this study, we introduced an improved edge detection method LAS based on the configuration of the generalised logistic function and the ratio of the vertical gradient and total horizontal gradient of the analytical signal amplitude. Unlike almost other edge enhancement methods that require a reduction to the pole or pseudogravity transformation prior to application, the LAS can be applied to the magnetic dataset directly. The disadvantage of the proposed method is that it is more sensitive to noise than some conventional methods that use only first-order derivatives of the field. Using an 
upward continuation filter, the noise effect can be reduced. The evaluation of the method comparative with other filters on both the synthetic and real magnetic data illustrate the capability and practicability for interpretation of magnetic data. The results show that the presented method can not only balance the amplitude responses from both shallow and deep sources, but also give a higher resolution, and can detect the source edges more clearly and precisely.

Acknowledgements. The authors would like to thank the editor Peter Vajda and Dr. Roman Pašteka for their very useful suggestions to improve the manuscript.

\section{References}

Ansari A. H., Alamdar K., 2011: A new edge detection method based on the analytic signal of tilt angle (ASTA) for magnetic and gravity anomalies. Iran. J. Sci. Technol., 35, 2, A2, 81-88, doi: 10.22099/IJSTS.2011.2131.

Birch C., 1999: A New Generalized Logistic Sigmoid Growth Equation Compared with the Richards Growth Equation. Ann. Bot., 83, 6, 713-723, doi: 10.1006/anbo.1999. 0877.

Chen A. G., Zhou T. F., Liu D. J., Zhang S., 2017: Application of an enhanced thetabased filter for potential field edge detection: a case study of the Luzong ore district. Chin. J. Geophys., 60, 2, 203-218, doi: 10.1002/cjg2.30039.

Chetty T. R. K., 2017: Proterozoic Orogens of India, A Critical Window to Gondwana. Chapter 4 ? The Central Indian Tectonic Zone. 211-265, doi : 10.1016/B978-0-12 -804441-4.00004-3.

Cooper G. R. J., 2014a: Reducing the dependence of the analytic signal amplitude of aeromagnetic data on the source vector direction. Geophysics, 79, 4, J55-J60, doi : 10.1190/geo2013-0319.1.

Cooper G. R. J., 2014b: The Automatic Determination of the Location and Depth of Contacts and Dykes from Aeromagnetic Data. Pure Appl. Geophys., 171, 9, 24172423, doi : 10.1007/s00024-014-0789-8.

Cooper G. R. J., Cowan D. R., 2006: Enhancing potential field data using filters based on the local phase. Comput. Geosci., 32, 10, 1585-1591, doi: 10.1016/j.cageo. 2006 .02 .016 .

Fairhead J. D., Williams S. E., 2006: Evaluating normalized magnetic derivatives for structural mapping. SEG Technical Program Expanded Abstracts, SEG 2006 New Orleans, 25, 1, 845?849, doi: 10.1190/1.2370388.

Ferreira F. J. F., Souza J., Bongiolo A. B. S., Castro L. G., 2013: Enhancement of the total horizontal gradient of magnetic anomalies using the tilt angle. Geophysics, 78, 3, J33-J41, doi : 10.1190/geo2011-0441.1. 
Florio G., Fedi M., Pasteka R., 2006: On the application of Euler deconvolution to the analytic signal. Geophysics, 71, 6, L87-L93, doi: 10.1190/1.2360204.

Ghosh G. K., 2016: Magnetic data interpretation for the source-edge locations in parts of the tectonically cctive transition zone of the Narmada-Son lineament in central India. Pure Appl. Geophys., 173, 2, 555-571, doi : 10.1007/s00024-015-1082-1.

Hsu S. K., Coppense D., Shyu C. T., 1996: High-resolution detection of geologic boundaries from potential field anomalies: An enhanced analytic signal technique. Geophysics, 61, 2, 373-386, doi: 10.1190/1.1443966.

Le-Huy M., Luu H. V., Cao T. D., 2001: Some modern methods of the interpretation aeromagnetic data applied for Tuan Giao region. Vietnam J. Earth Sci., 23, 3, 207-216, doi: 10.15625/0866-7187/23/3/11333, (in Vietnamese with English summary).

Le-Huy M., Luu H. V., Cao T. D., 2002: Using the maximum horizontal gradient vector to interpret magnetic and gravity data in Vietnam. Vietnam J. Earth Sci., 24, 1, 6780, doi : 10.15625/0866-7187/24/1/11380, (in Vietnamese with English summary).

Li X., Pilkington M., 2016: Attributes of the magnetic field, analytic signal, and monogenic signal for gravity and magnetic interpretation. Geophysics, 81, 6, 79?86, doi : 10.1190/geo2015-0697.1.

Ma G., Liu C., Li L., 2014: Balanced horizontal derivative of potential field data to recognize the edges and estimate location parameters of the source. J. Appl. Geophys., 108, 12-18, doi: 10.1016/j.jappgeo.2014.06.005.

Malkina-Pykh I. G., Pykh, Y. A., 2013: The Method of Response Function in Psychology and Sociology. Southampton Boston: WIT Press, p. 69.

Miller H. G., Singh V., 1994: Potential field tilt-a new concept for location of potential field sources. J. Appl. Geophys, 32, 213-217, doi : 10.1016/0926-9851 (94)90022-1.

Mohanty S., 2012: Spatio-temporal evolution of the Satpura Mountain Belt of India: A comparison with the Capricorn Orogen of Western Australia and implication for evolution of the supercontinent Columbia. Geosci. Front., 3, 3, 241-267, doi : 10.1016/ j.gsf.2011.10.005.

Nasuti Y., Nasuti A., 2018: NTilt as an improved enhanced tilt derivative filter for edge detection of potential field anomalies. Geophys. J. Int., 214, 1, 36-45, doi : 10.1093/ gji/ggy 117.

Nelder J. A., 1961: The Fitting of a Generalization of the Logistic Curve. Biometrics, 17, 1, 89-110, doi : 10.2307/2527498.

Nguyen H. T. T., Do T. D., Le-Huy M., 2017: Application of directional derivative method to determine boundary of magnetic sources by total magnetic anomalies. Vietnam J. Earth Sci., 39, 4, 360-375, doi: 10.15625/0866-7187/39/4/10731.

Paoletti V., Fedi M., Florio G., Supper R., Rapolla A., 2004: The new integrated aeromagnetic map of the Phlegrean Fields volcano and surrounding areas. Ann. Geophys.Italy, 47, 5, 1569-1580, doi : 10.4401/ag-3360.

Pham L. T., Le-Huy M., Oksum E., Do T. D., 2018: Determination of maximum tilt angle from analytic signal amplitude of magnetic data by the curvature-based method, Vietnam J. Earth Sci., 40, 4, 354-366, doi: 10.15625/0866-7187/40/4/13106. 
Pham L. T., Oksum E., Do T. D., 2019: Edge enhancement of potential field data using the logistic function and the total horizontal gradient, Acta Geod. Geophys., 54, 1, 143-155, doi : 10.1007/s40328-019-00248-6.

Project Crumansonata, 1995: Geoscientific studies of the Son-Narmada-Tapti lineament zone. Calcutta, Geological Survey of India, Special publication, 10, pp. 371.

Rao D. B., Babu N. R., 1991: A rapid method for three-dimensional modeling of magnetic anomalies. Geophysics, 56, 11, 1729-1737, doi: 10.1190/1.1442985.

Roest W. R., Verhoef J., Pilkington M., 1992: Magnetic interpretation using the 3-D analytic signal. Geophysics, 57, 1, 116-125, doi: 10.1190/1.1443174.

Tran D. T., Nguyen M. Q., 2017: Eruptive-volcanic-basalt structures in the Truong SaSpratly Islands and adjacent areas from interpreting gravity and magnetic data. Vietnam J Earth Sci, 39, 1, 1-13, doi: 10.15625/0866-7187/39/1/9167.

Saibi H., Azizi M., Mogren S., 2016: Structural Investigations of Afghanistan Deduced from Remote Sensing and Potential Field Data. Acta Geophys., 64, 4, 978-1003, doi : 10.1515/acgeo-2016-0046.

Saleh S., Pašteka R., 2012: Applying the regularized derivatives approach in Euler deconvolution and modeling geophysical data to estimate the deep active structures for the northern Red Sea Rift region, Egypt. Contrib. Geophys. Geod., 42, 1, 25-61, doi: $10.2478 / v 10126-012-0003-x$.

Salem A., Ravat D., 2003: A combined analytic signal and Euler method (AN-EUL) for automatic interpretation of magnetic data. Geophysics, 68, 6, 1952-1961, doi: $10.1190 / 1.1635049$.

Verduzco B., Fairhead J. D., Green C. M., MacKenzie C., 2004: New insights into magnetic derivatives for structural mapping. Lead. Edge, 23(2), 116-119, doi: 10.1190/ 1.1651454 .

Wijns C., Perez C., Kowalczyk P., 2005: Theta map: edge detection in magnetic data. Geophysics, 70, 4, 39-43, doi: 10.1190/1.1988184.

Yao Y., Huang D., Yu X., Chai B., 2015: Edge interpretation of potential field data with the normalized enhanced analytic signal. Acta Geod. Geophys., 51, 1, 125-136, doi : $10.1007 / \mathrm{s} 40328-015-0120-x$.

Zhang X., Yu P., Tang R., Xiang Y., Zhao C. J., 2014: Edge enhancement of potential field data using an enhanced tilt angle. Explor. Geophys., 46, 3, 276-283, doi: 10.1071/EG13104. 